\title{
Comment
}

Neuro epidemiology

Neuroepidemiology 2014;42:184-185

DOI: $10.1159 / 000360431$

\section{The Global Epidemiology of Hereditary Ataxia and Spastic Paraplegia: What Are the Messages?}

\section{Mark Braschinsky}

Tartu University Clinics, Tartu, Estonia

Hereditary spastic paraplegias (HSP) and hereditary cerebellar ataxias (HCA) form a group of clinically and genetically heterogeneous disorders. If HSP is clinically characterized by the key features of progressive spasticity and hyperreflexia of the lower limbs [1], then the leading clinical presentation of HCA is the cerebellar syndrome [2]. In recent years there have been an increasing number of publications describing the prevalence of HSP and HCA. The results varied considerably, depending on different factors, including big differences in methodological approach. Within these reports both ends of the spectrum were represented: some authors have chosen to report on isolated forms of a particular disease, for instance concentrating on autosomal dominant forms [3], others preferred to investigate both entities - HSP and HCA with all the subforms - at the same time [4]. Some earlier studies calculated prevalence rates based on clinical diagnosis [5], more recent ones took into consideration the genetic background [6]. Furthermore, the vast majority if not all of these studies were performed locally in isolated geographic regions or individual countries. So far we lacked information about the global prevalence of HSP and HCA.

In the current issue of Neuroepidemiology Ruano et al. [7] publish their systematic review and meta-analysis of the literature in order to try to fill the gap mentioned above. The authors searched several databases (dated 1983-2013) for studies performed in welldefined populations and geographic regions. The publications they found were assessed for their quality and compliance with the criteria of the review. For the final analysis the authors included 22 publications. Based on the latter, prevalence rates were calculated for separate genetic forms of HSP and HCA, stressing the most frequent ones of both disorders. Results proved to be highly variable, which is in concordance with an overview of the literature one might undertake in order to get 'the whole picture'.

Interestingly, Ruano et al. [7] also presented an estimated prevalence for the general population to have either HSP or HCA. It is debatable if the latter approach to view both entities - HSPs and HCAs - as one group of disorders is ultimately justified. There are several arguments against it. Despite their heritability, these disorders represent different nosologic entities. Even though some overlap may exist, the clinical syndromological presentation of the diseases is separable: in case of HSP the leading syndrome is pyramidal, whereas spasticity and hyperreflexia of the lower limbs are by far the most dominating obligatory presentation; HCA's leading syndrome is cerebellar. Furthermore, when we look at the two separate groups of diseases (HSP vs. HCA), suggested neuropathologic mechanisms for each of them are rather different. In HSP it is mainly distal axonal degeneration of the longest tracts within the central nervous system and in case of HCA it is neuronal loss in the cerebellum. But, having said that, we must acknowledge, that despite recent advances in our understanding of the neuropathology and proposed pathogenetic mechanisms of HSP and HCA, we are still lacking a great deal of knowledge. Further discoveries might change our understanding substantially and lead to new grounds for classification of both entities. But until that, it might be more reasonable to keep both hereditary groups of disorders separated for such analyses.

Nevertheless, the undertaken review of global prevalence rates of HSP and HCA emphasized a number of issues in need of consideration for future studies.

Further genetic research is needed, to fill the large gap stressed by Ruano et al. [7]: according to the present study, globally up to $92 \%$ of patients with either HSP or HCA do not have a genetic confirmation of clinically established diagnosis!

The quality of acquired epidemiologic data depends on the selection of the most appropriate methodological approach. There might be several different approaches to reach the same goal appropriately, but in order to have comparable results, the standardization of methods is vital. As the present publication showed, different groups of investigators use different methods in this field. It might be suggested that for further studies we need a consensus resulting in guidelines on the performance of such studies. These guidelines should take into consideration the specificity of this area of neurology.

And last but not least, despite the heading of the present publication, stating that this study represents 'the global' prevalence of both disorders, this statement might be arguable as well. This systematic review and meta-analysis used an acquired database from only 16 countries. Can we use these 16 countries to conclude that the authors found real global prevalence rates? Probably not. But this study definitely is the first of its kind and the largest one in this area. It clearly systematizes our impression of the prevalence of HSP and HCA in those regions of the world, where it has been investigated in a methodologically acceptable way. What we can really conclude is that there is a clear need for expanding the geographic representation of prevalence studies of HSP and HCA, preferably separately for both diseases.

\section{KARGER}

E-Mail karger@karger.com

www.karger.com/ned
C 2014 S. Karger AG, Base

0251-5350/14/0423-0184\$39.50/0
Mark Braschinsky

Tartu University Clinics

2 L. Puusepp Street

EE-51014 Tartu (Estonia)

E-Mail mark.braschinsky@ kliinikum.ee 


\section{References}

1 Tallaksen CM, Dürr A, Brice A: Recent advances in hereditary spastic paraplegia. Curr Opin Neurol 2001;14:457-463.

2 Finsterer J: Ataxias with autosomal, X-chromosomal or maternal inheritance. Can J Neurol Sci 2009;36:409-428.

3 McMonagle P, Webb S, Hutchinson M: The prevalence of 'pure' autosomal dominant hereditary spastic paraparesis in the island of Ireland. J Neurol Neurosurg Psychiatry 2002;72:43-46.

4 Coutinho P, Ruano L, Loureiro JL, et al: Hereditary ataxia and spastic paraplegia in Portugal: a population-based prevalence study. JAMA Neurol 2013;70:746-755.
5 Leone M, Bottacchi E, D’Alessandro G, et al: Hereditary ataxias and paraplegias in Valle d'Aosta, Italy: a study of prevalence and disability. Acta Neurol Scand 1995;91:183-187.

6 Braschinsky M, Lüüs S-M, Gross-Paju K, et al: The prevalence of hereditary spastic paraplegia and the occurrence of SPG4 mutations in Estonia. Neuroepidemiology 2009;32:89-93.

7 Ruano L, Melo C, Silva MC, Coutinho P: The global epidemiology of hereditary ataxia and spastic paraplegia: a systematic review of prevalence studies. Neuroepidemiology 2014;42:174-183. 